\title{
Kennedy D, Hwang P. Rhinology: Diseases of the Nose, Sinuses, and Skull Base
}

\author{
New York: Thieme medical Publishers; 2012. ISBN 978-1-60406-060-7
}

\author{
Samy Elwany
}

Published online: 7 October 2012

(c) Springer-Verlag Berlin Heidelberg 2012

Rhinology: Diseases of the Nose, Sinuses, and Skull Base is a worthwhile well-organized textbook written by 134 authors, and edited by D. Kennedy and P. Hwang. This single volume textbook features an up-to-date review of sinonasal anatomy and physiology, as well as medical and surgical aspects of diseases of the nose, sinuses, and related skull base.

The text is 776 pages in length and features contributions of many of the leading experts in the field. A wellnarrated DVD is included with the book. The textbook has 54 chapters, divided into 4 sections covering a wide array of topics. Each chapter ends with an excellent recent list of references. Almost all chapters include many high-quality pertinent figures and color illustrations. All clinical and histopathological pictures are also in full color.

The first section (4 chapters) deals with sinonasal anatomy and physiology and also includes a well-illustrated chapter on imaging of paranasal sinuses and skull base, and another chapter on objective measures of nasal functions. This last chapter, together with the chapters on mucociliary clearance and olfaction, is imperative for research in nasal physiology and clinical outcome studies.

The second section (16 chapters) discusses medical aspects of inflammatory sinonasal diseases and includes valuable information for the clinicians and researchers as well. The chapter on diagnosis of rhinosinusitis is nicely illustrated. There is also a whole chapter on principles of allergy skin testing and immunotherapy and another attention-grabbing chapter on management of recalcitrant rhinosinusitis.
The third section (19 chapters) concentrates on surgical aspects of sinonasal diseases and is complemented by the accompanying DVD. This section focuses on diseases that are treated primarily surgically. It also includes chapters on sinonasal trauma and epistaxis. The chapters on office rhinology and instrumentation for sinus surgery are basically important for junior rhinologists and ENT surgeons. Contrarily, the chapter on surgical navigation and intraoperative imaging is valuable for experienced rhinologists and skull base surgeons.

The last section (14 chapters) deals with endoscopic surgery of the anterior and central skull base and is especially interesting for experienced rhinologists. In addition, the section includes also endoscopic approaches to the odontoid, clivus, and posterior fossa.

The accompanying DVD includes 21 videos narrated by David Kennedy in a consistent manner. The videos are of good quality and demonstrate the various surgical procedures described in the text. The first video, however, is on anatomy of paranasal sinuses.

Overall, Rhinology: Diseases of the Nose, Sinuses, and Skull Base is a welcome addition to the libraries of novice and senior otorhinolaryngologists and, of course, rhinologists. The book is an important multimedia resource for experienced surgeons and young trainees. It includes the present-time experience of many of the world top rhinologists, and it is definitely an excellent reserve of a firm knowledge base of the field of rhinology and related skull base surgery.

S. Elwany $(\square)$

Alexandria, Egypt

e-mail: samyelwany@msn.com 